\title{
Knowledge, Attitude, and Beliefs Regarding Convalescent Plasma Donation among COVID-19-recovered Patients at a Tertiary Care Center in India
}

\author{
Parveen Kumar ${ }^{1} \odot$, Rangdon Dor Sangma², Lubna Mohammedrafik Nerli ${ }^{3}$, Disha Alkeshbhai Vasavada ${ }^{4}$, \\ Krishna Manharbhai Mayani ${ }^{5}$, Deepak Sachidanand Tiwari ${ }^{6}$
}

\section{Abstract}

Background: Convalescent plasma is gathered from a possible human source of specific antibodies. Convalescent plasma is released as an "off-label" therapy for novel coronavirus 2019 (COVID-19). Amid the national shortage of this blood product, it is critically important that those who have recovered from COVID-19 donate plasma.

Aim: The current study aimed to assess the knowledge and attitude of COVID-19 recovered patients toward convalescent plasma donation. Method: A cross-sectional study was carried out among patients discharged after recovery from COVID-19 at a tertiary care center from August 2020 to December 2020. Participants were approached 30 to 45 days after discharge from the hospital and requested to complete a semistructured pro forma containing the following sections: (1) Demographic details; (2) Knowledge about Convalescent Plasma Questionnaire; (3) Attitude toward Convalescent Plasma Questionnaire; and (4) Reasons for not donating plasma. Following this, education was given to participants about plasma donation.

Result: A total of 400 participants were included in the study of age 18 to 60 years, out of them $42.75 \%$ donated plasma and $57.25 \%$ did not donate. Participants have good knowledge about their blood group, the requirement of blood group matching for plasma donation, and the use of convalescent plasma therapy. Plasma donors have a more positive attitude toward donation. Among non-donors, $47.8 \%$ of participants were unaware, while $42 \%$ did not know about fitness for plasma donation. Non-donors have the misbelief that plasma donation temporarily weakened the individual and those who donate plasma can contract a disease. Slight negative correlation between "knowledge" and "attitude" toward plasma donation' scores on the "Pearson correlation test" $(r=0-0.142, p=0.005)$.

Conclusion: Donors have a more positive attitude toward plasma donation. The idea of voluntary plasma donation can be intensively promoted by improving knowledge and awareness through education and specific campaigns.

Keywords: Attitude, Blood products, COVID-19, Knowledge, Plasma, SARS-CoV-2.

Indian Journal of Private Psychiatry (2020): 10.5005/jp-journals-10067-0068
\end{abstract}

\section{INTRODUCTION}

Novel coronavirus 2019 (COVID-19) belonging to severe acute respiratory syndrome coronavirus 2 (SARS-CoV-2), a positive-sense, single-stranded RNA virus has emerged as a global pandemic that has no proven treatment. Due to this, clinicians are put in a serious therapeutic dilemma requiring therapies that could help to reduce patient mortality. Convalescent plasma therapy might have some therapeutic potential amid the therapeutic uncertainties. ${ }^{1}$ Blood, plasma, serum, and immunoglobulin are included in convalescent blood products. These products are gathered from a possible human source of specific antibodies, i.e., the patient who has already recovered from an infection. ${ }^{2}$ Previously convalescent plasma therapy has been efficacious in other virus-borne infections. The World Health Organization recommended the use of convalescent plasma from recovered patients for empirical treatment during the Ebola outbreak, ${ }^{3}$ influenza A virus subtype H1N1 pandemic, ${ }^{4}$ Middle East respiratory syndrome coronavirus, ${ }^{5}$ and SAR infections viruses. ${ }^{6}$

The protection from COVID-19 infection or disease has yet to be directly correlated with levels of circulating antibodies against SARS-CoV-2, ${ }^{7}$ providing virus-neutralizing antibodies in the form of convalescent plasma. ${ }^{8}$ The accepted mechanism of convalescent plasma therapy is clearance of viremia happening around 10 to 14 days after infection. ${ }^{9}$ The early use of convalescent
1-4,6Department of Psychiatry, MP Shah Govt Medical College,
Jamnagar, Gujarat, India
5epartment of IHBT, MP Shah Govt Medical College, Jamnagar,
Gujarat, India

Corresponding Author: Lubna Mohammedrafik Nerli, Department of Psychiatry, MP Shah Govt Medical College, Jamnagar, Gujarat, India, Phone: +91 9624731989, e-mail: lubna.nerli@gmail.com

How to cite this article: Kumar P, Sangma RD, Nerli LM, et al. Knowledge, Attitude, and Beliefs Regarding Convalescent Plasma Donation among COVID-19-recovered Patients at a Tertiary Care Center in India. Ind J Priv Psychiatry 2020;14(2):80-84.

Source of support: Nil

Conflict of interest: None

plasma therapy may be considered as an adjuvant for critically ill COVID-19 patients despite significant limitations, such as lack of availability, paucity of compelling clinical evidence advocating it, and standardization of this treatment method and its use.

Convalescent plasma therapy is released as an "off-label" therapy along with remdesivir and tocilizumab by the Drug Controller General of India (DCGI). As per DCGI, plasma will only be collected 
from recovered fit patients of the age-group 18 to 60 years. Before collection of plasma, an individual donor will be tested for adequate levels of antibodies, hemoglobin level, and blood group. ${ }^{10}$ Due to increase in the number of cases and to help patients battling the virus, there is a continuous requirement of availability for convalescent plasma. As there is a national shortage of this potentially lifesaving blood product, the Red Cross urges eligible COVID-19 survivors to donate plasma for helping current COVID-19 patients. It is of utmost importance that those who have recovered from COVID-19 donate plasma now to help patients in need. ${ }^{11}$

The result of previous studies shows that higher knowledge develops a more favorable attitude toward the donation of convalescent plasma. The closer understanding of psychological factors, such as the motivation, knowledge, and attitudes of potential convalescent plasma donors, is critical to the success of their recruitment. ${ }^{12}$

The current literature is deficient in the knowledge and attitude of people toward convalescent plasma donation for the treatment of COVID-19 patients. Therefore, the current study was conducted to assess the knowledge and attitude of COVID-19 recovered patients toward convalescent plasma donation at a tertiary care center in India.

\section{Methods and Materials Methodology}

A cross-sectional study was carried out among patients discharged after recovery from COVID-19 at a tertiary care center from August 2020 to December 2020. All the participants were approached 30 to 45 days after discharge from the hospital and requested to complete a semi-structured pro forma containing the following sections: (1) Demographic details; (2) Knowledge about Convalescent Plasma Questionnaire; (3) Attitude toward Convalescent Plasma Questionnaire; and (4) Reasons for not donating plasma. The questionnaire was pretested on 25 participants of similar gender and age-group and the difficulties faced by them in some of the items were rephrased. Following this, education was given to participants about who can donate plasma, age-group for plasma donation, laboratory reports done at the time of donation, duration after which plasma can be donated, procedure of plasma donation, places where plasma can be donated, and risk of contracting a disease after plasma donation. In the end, the queries of patients regarding plasma donation were answered. Participants who consented were included in the study. The ethical approval was taken from the institutional ethical committee.

\section{Sample Size Calculation}

The sample size required for the current study was calculated using Epilnfo software. The sample size for the current study was estimated to be 386 by using a $95 \%$ confidence interval and 5\% absolute precision.

\section{Materials}

\section{Demographic Details}

Demographic details included parameters like age, gender, profession, working area, marital status, living alone or with family, and their education status.

\section{Knowledge about Convalescent Plasma Questionnaire}

Ten-item Knowledge about Convalescent Plasma Questionnaire was developed on the basis of following factors: awareness of their blood group; donated blood/blood products in past; necessity to match blood group for plasma therapy; risk of transmitting COVID-19 virus to the recipient of plasma therapy; procedure of donating plasma, who can get benefit from convalescent COVID plasma therapy; is convalescent plasma therapy first time used for the treatment of any infection; minimum age for convalescent COVID plasma donation; donor with comorbidities, like diabetes mellitus (DM) and hypertension (HTN), can donate convalescent plasma; and can a donor donate convalescent plasma more than once. Knowledge statements were scored as "yes" and "no". The correct response was given as " 1 " and incorrect response as " 0 ", with a total score ranges from 0 to 10 . The questionnaire was derived from Chauhan et al. Knowledge and attitude about blood donation Questionnaire. ${ }^{13}$ This scale showed adequate reliability with a Cronbach's alpha internal consistency coefficient of 0.86 for the current sample.

\section{Attitude toward Convalescent Plasma Donation Questionnaire}

Twelve-item Attitude toward Convalescent Plasma Questionnaire was developed on the basis of following factors: plasma donation saves lives and it is a moral activity, every person infected with COVID-19 infection should donate plasma, best way to donate plasma is voluntary non-remunerated, best way to donate plasma is at the request of relatives, every person should always disclose correct information about his/her health before donating plasma, people who donate plasma should receive something in exchange, people who donate plasma can contract a disease, and donating blood plasma temporarily weakened the individual. Attitudinal statements were scored on a 5 point Likert scale: $1=$ strongly agree, 2 = agree, $3=$ do not know, $4=$ disagree, and $5=$ strongly disagree, with a higher score representing negative attitude. The questionnaire was derived from Chauhan et al. Knowledge and attitude about blood donation Questionnaire. ${ }^{13}$ This scale showed adequate reliability with a Cronbach's alpha internal consistency coefficient of 0.73 for the current sample.

\section{Reasons for Not Donating Plasma Questionnaire}

Multiple response questionnaire containing different questions, such as fear of needle pain, fear of becoming weak, fear of contracting a disease, no time for plasma donation, fear of discovering some unknown disease on testing, no one ever asked me to donate plasma, and unawareness about places of plasma donation in the city, were used for assessing reasons for not donating plasma.

\section{Statistical Analysis}

All the collected data were tabulated in Microsoft Excel and analyzed by using statistical software Statistical Package for Social Sciences, version 20.0. Frequencies and percentages were computed for the sociodemographic and knowledge about convalescent plasma. The data not following normal distribution were assessed by Kolmogorov-Smirnov test. Mann-Whitney $U$ test was applied to compare attitude of plasma "donors" and "non-donors" participants. Percentage tabulation was used for "reasons for not donating plasma". Pearson correlation test was used for relation between "attitude toward plasma donation" and "knowledge about plasma donation" scores. $p<0.05$ was considered as statistically significant.

\section{Results}

A total of 400 participants were included in the study of age 18 to 60 years, with a mean age of $42.27 \pm 11.22$ years. Out of them, 244 
(61\%) were males and $156(39 \%)$ were females. The majority of them [320 (80\%)] belong to the Hindu religion.

Out of 400 participants, 171 (42.75\%) donated plasma and 229 (57.25\%) not donated. Out of plasma donors, $48.2 \%$ donated for replacement and $51.8 \%$ have voluntary donation.

Table 1 shows that participants have good knowledge about their blood group, the requirement of blood group matching for plasma donation, minimum age for plasma donation, risk of transmitting COVID-19 due to plasma therapy, use of convalescent plasma therapy, and about numbers of times plasma can be donated.

Table 2 shows that plasma donors have the positive attitude that plasma donation saves lives, every person should donate blood, and best way to donate plasma is voluntary non-remunerated. While non-donors believe that plasma donation temporarily weakened the individual, who donate plasma can contract a disease and in favor of paid donation. No difference was observed among plasma donors and non-donors, when it is requested by relatives and donation is a moral activity.

Table 3 shows that female participants with a family history of COVID infection have a statistically significant positive attitude toward plasma donation, which was denoted by Mann-Whitney $U$ test. Young participants (18-30 years) have a statistically significant higher positive attitude toward plasma donation, which was denoted by Kruskal-Wallis test $(H=33.219, p<0.001)$.

Table 3: Comparison of attitude toward plasma donation of different demographical variables $(n=400)$

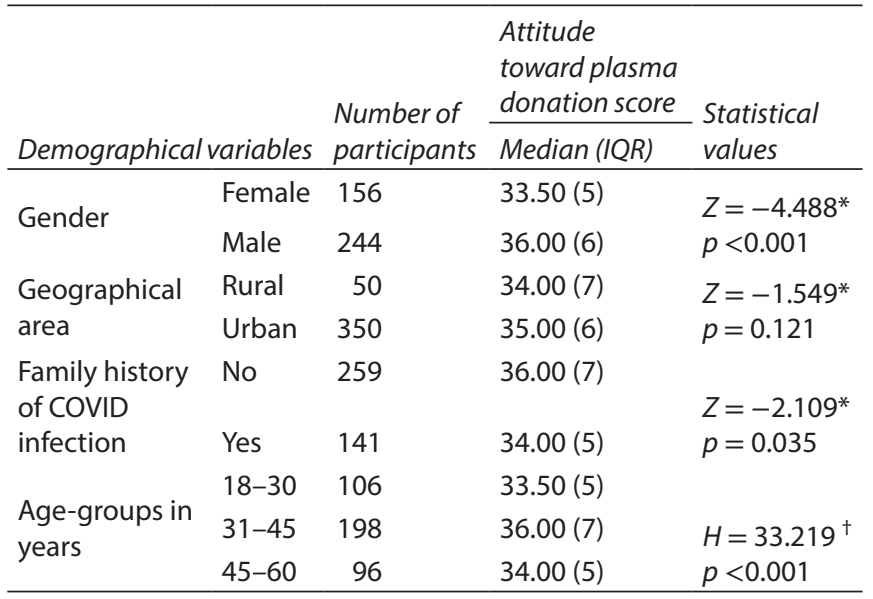

*Mann-Whitney $U$ test;

${ }^{\dagger}$ Kruskal-Wallis test

Table 1: Response of participants on different Knowledge about Convalescent Plasma Questionnaire $(n=400)$

\begin{tabular}{|c|c|c|c|}
\hline \multirow[b]{2}{*}{ SI. No. } & \multirow[b]{2}{*}{ Knowledge about Convalescent Plasma Questionnaire } & \multicolumn{2}{|c|}{ Response of participants N (\%) } \\
\hline & & Yes & No \\
\hline 1 & Are you aware of your blood group? & $364(91.0)$ & $36(09.0)$ \\
\hline 2 & Have you donated blood/blood products in past? & $175(43.7)$ & $225(56.3)$ \\
\hline 3 & Is it necessary to match blood group for plasma therapy? & $294(73.5)$ & $106(26.5)$ \\
\hline 4 & Is there risk of transmitting COVID-19 virus to the recipient of plasma therapy? & $78(19.5)$ & $322(80.5)$ \\
\hline 5 & Do you think donating plasma is a long procedure? & $136(34.0)$ & $264(66.0)$ \\
\hline 6 & Convalescent plasma therapy should be used only for severe to fatal cases of COVID-19? & $288(72.0)$ & $112(28.0)$ \\
\hline 7 & Is convalescent plasma therapy first time used for treatment of any infection? & $94(24.5)$ & $306(76.5)$ \\
\hline 8 & The minimum age for convalescent COVID plasma donation is 18 years. & $331(82.7)$ & $69(17.3)$ \\
\hline 9 & Donor with comorbidities like DM and HTN can donate convalescent plasma. & $182(45.5)$ & $218(54.5)$ \\
\hline 10 & Can a donor donate convalescent plasma more than once? & $301(75.3)$ & $99(24.7)$ \\
\hline
\end{tabular}

Table 2: Comparison of attitude toward plasma donation among plasma donors and non-donors $(n=400)$

\begin{tabular}{|c|c|c|c|c|c|c|c|}
\hline \multirow[b]{3}{*}{ SI. No. } & \multirow[b]{3}{*}{ Attitude toward plasma donation Questionnaire } & \multicolumn{4}{|c|}{ Plasma donated } & \multirow{2}{*}{\multicolumn{2}{|c|}{$\begin{array}{l}\text { Test statistics: } \\
\text { Mann-Whitney U test }\end{array}$}} \\
\hline & & \multicolumn{2}{|c|}{ No } & \multicolumn{2}{|c|}{ Yes } & & \\
\hline & & Median & $I Q R$ & Median & $I Q R$ & $Z$ & $p$ \\
\hline 1 & I think plasma donation saves lives. & 2 & 1 & 2 & 1 & -2.323 & 0.02 \\
\hline 2 & I think plasma donation is a moral activity & 2 & 1 & 1 & 1 & -1.012 & 0.31 \\
\hline 3 & I think every person infected with COVID-19 infection should donate blood. & 2 & 1 & 2 & 2 & -5.063 & $<0.001$ \\
\hline 4 & $\begin{array}{l}\text { I think people having knowledge on COVID plasma donation are ready to } \\
\text { donate plasma. }\end{array}$ & 3 & 2 & 2 & 1 & -5.849 & $<0.001$ \\
\hline 5 & In my opinion, the best way to donate plasma is voluntary non-remunerated. & 2 & 0 & 2 & 1 & -2.749 & 0.005 \\
\hline 6 & $\begin{array}{l}\text { I think every person should always disclose correct information about his/her } \\
\text { health before donating plasma. }\end{array}$ & 1 & 1 & 1 & 1 & -2.809 & 0.005 \\
\hline 7 & In my opinion, the best way to donate plasma is at the request of relatives. & 4 & 1 & 4 & 1 & -0.267 & 0.789 \\
\hline 8 & In my opinion, the best way to donate plasma is paid donation. & 4 & 0 & 5 & 1 & -6.825 & $<0.001$ \\
\hline 9 & I think people who donate plasma should receive something in exchange. & 4 & 1 & 4 & 2 & -1.661 & 0.097 \\
\hline 10 & I think people who donate plasma can contract a disease. & 4 & 1 & 4 & 1 & -4.533 & $<0.001$ \\
\hline 11 & I think people who donate blood plasma are temporarily weakened. & 4 & 2 & 4 & 2 & -3.578 & $<0.001$ \\
\hline 12 & I am donating plasma to get free investigations. & 4 & 1 & 4 & 2 & -2.906 & 0.004 \\
\hline
\end{tabular}




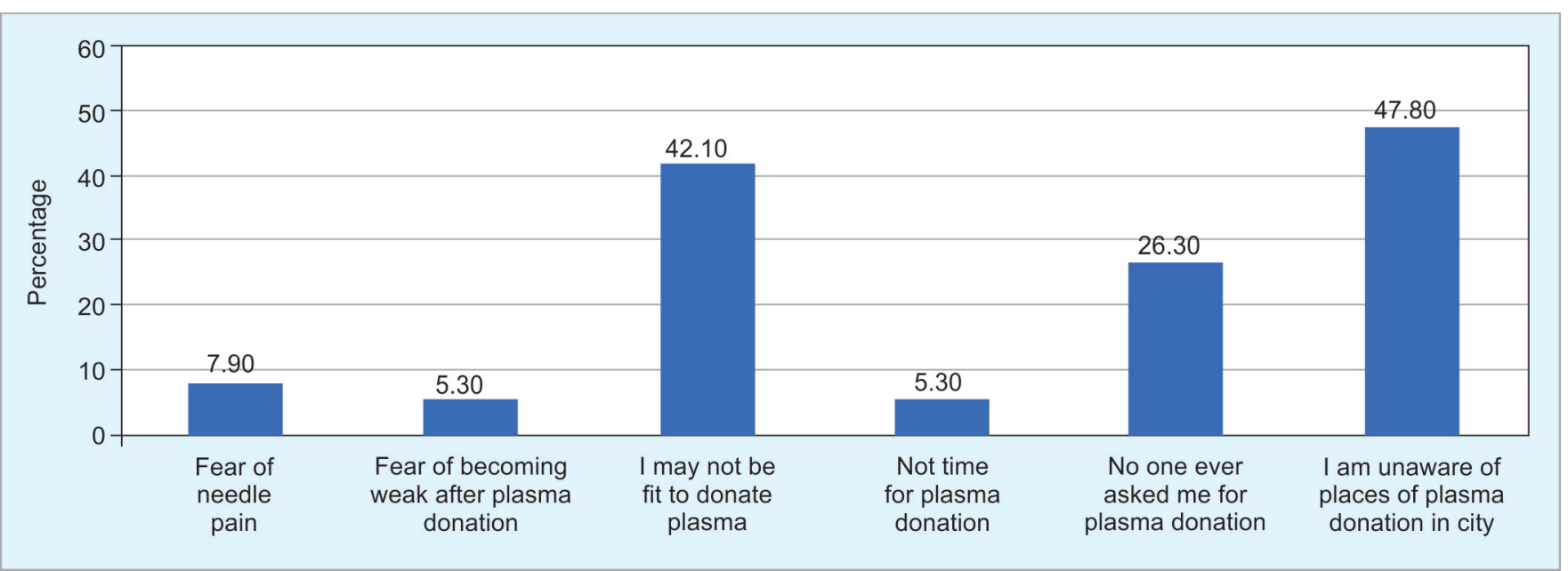

Fig. 1: Reasons of participants for not donating plasma $(n=229)$

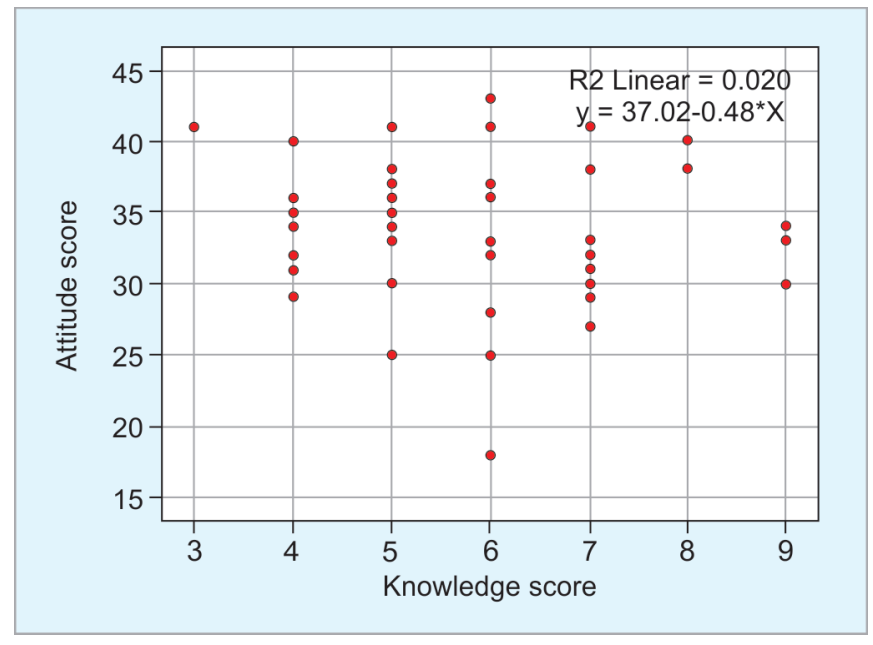

Fig. 2: Relation between attitude toward plasma donation and knowledge about plasma donation scores

Figure 1 shows that $47.8 \%$ of participants were unaware about plasma donation, while $42 \%$ did not know about fitness for plasma donation. Some participants have a misconception that plasma donation leads to weakness and needle pain.

Figure 2 shows that there is a slight negative correlation between "attitude toward plasma donation" and "knowledge about plasma donation" scores, which is found to be statistically significant as denoted by the Pearson correlation test $(r=-0.142$, $p=0.005)$. This means that participants with higher knowledge have a positive attitude toward plasma donation.

Out of participants with a family history of COVID infection, $53.2 \%$ donated plasma, which is statistically, significantly higher as compared to $37.1 \%$ among participants without a family history of COVID infection, which is denoted by the chi-square test $\left(x^{2}=9.700\right.$, $p=0.002$ ).

\section{Discussion}

In India, convalescent plasma services and designated centers for donation are provided by the government health services network. Maintaining a stable and adequate plasma supply is a huge challenge.

The current study observed that participants have good knowledge about their blood group, the requirement of blood group matching for plasma donation, minimum age for plasma donation, risk of transmitting COVID-19 due to plasma, use of Convalescent Plasma therapy, and about numbers of time plasma can be donated. Also greater knowledge was linked to a positive attitude toward plasma donation. Saeed et al. also observed that adequate knowledge level was significantly linked with a good attitude and practice toward plasma donation. ${ }^{14}$ Beyene et al. reported that the good knowledge and favorable attitude were associated with blood donation practice. ${ }^{15}$ However, Wiwanitkit et al. observed that greater knowledge about blood donation does not lead to donation and there is a need for specific campaigns to improve actual voluntary donation. ${ }^{16}$

The current study observed that $42.75 \%$ of participants donated plasma. While Saeed et al. in Pakistan observed that $28.5 \%$ of participants donated plasma. ${ }^{14}$ Beyene et al. observed that only $17 \%$ of study participants ever donated blood, of which $14.6 \%$ of them donated for replacement and less than $2 \%$ were voluntary donors. ${ }^{15}$ It was observed that by developing a pleasant attitude, listening to their worries, complaints, and suggestions of donor's fosters a better donor for blood donation. ${ }^{17}$ Higher prior donation frequency, convenient place to donate, having a good donation experience, feeling a responsibility to help others, and higher empathetic concern were also associated with a high intention to return. ${ }^{18}$

The current study observed that female participants with a family history of COVID infection and 18 to 30 years age-group have a more positive attitude toward plasma donation. While Shenga et al. not observed any significant correlation of gender and age, but educational level and family income were strong indicators for the probability of someone's donating blood. ${ }^{19}$ These results can be due to difference in the level of knowledge and experience of past donations.

The current study did not observed any attitude difference for the requirement of something in exchange for plasma donation, but non-donors believe that paid donation might improve donation. Shenga et al. observed that $20 \%$ of participants prefer some kind of nonmonetary benefit in the form of certificates of recognition and 
recommended incentive can be used in enhancing the effectiveness of blood donation campaigns. ${ }^{19}$

The current study observed various reasons were given by non-donor participants for not having donated plasma. Participant unawareness about plasma donation and deficit in knowledge about fitness for plasma donation linked to the low practice of plasma donation. Saeed et al. observed that fear of getting involved in some health issues is associated with the low donation rate. No one ever asked me for plasma donation is also reported by $26 \%$ of participants. ${ }^{14}$ Dubey et al. reported that $40.75 \%$ of blood nondonor's reason was "no one asked them to give blood". Misbelief related to blood donation also a reason for non-donation. ${ }^{17}$ Fear of hospitals, lack of awareness, religious traditions, and fear of needles and pain were also reasons for not donating blood. ${ }^{20,21}$ These factors can be overcome by promoting knowledge and awareness through advertising in media to keep the topic alive in the minds of the general public. The idea of voluntary plasma donation also needs to be intensively promoted.

The current study addresses many concerns about plasma donation. However, the study is limited by a cross-sectional design, the longitudinal and experimental studies are required to better elucidate causality. The study contains self-reported data that could be biased in the direction of both overreporting and underreporting. Factors, like religiosity, educational background, and experiences of past blood or blood product donation, were not included in this study, which could have an effect on plasma donation attitude. The study results are compared with blood donation studies due to the lack of literature about plasma donation among the general public.

\section{Conclusion}

Greater knowledge about plasma was liked to a positive attitude toward plasma donation. Participant unawareness about plasma donation and deficit in knowledge about fitness for plasma donation were linked to the low practice of plasma donation. These factors can be overcome by promoting knowledge and awareness through education among the general public.

\section{ORCID}

Parveen Kumar (1) https://orcid.org/0000-0002-8724-8433

Lubna Mohammedrafik Nerli i] https://orcid.org/0000-0002-2178-

4364/print

Krishna M Mayani (1) https://orcid.org/0000-0002-9230-4671

\section{References}

1. Bakhtawar N, Usman M, Khan MMU. Convalescent plasma therapy and its effects on COVID-19 patient outcomes: a systematic review of current literature. Cureus 2020;12(8):e9535. DOI: 10.7759/cureus.9535.

2. Marano G, Vaglio S, Pupella S, et al. Convalescent plasma: new evidence for an old therapeutic tool? Blood Transfus 2016;14(2):152157. DOI: 10.2450/2015.0131-15.

3. Griensven JV, Edwards T, de Lamballerie X, et al. Evaluation of convalescent plasma for Ebola virus disease in Guinea. New Engl J Med 2016;374(1):33-42. DOI: 10.1056/NEJMoa1511812.

4. Hung IF, To KK, Lee CK, et al. Convalescent plasma treatment reduced mortality in patients with severe pandemic influenza $A$
(H1N1) 2009 virus infection. Clin Infect Dis 2011;52(4):447-456. DOI: $10.1093 /$ cid/ciq106.

5. The WHO MERS-CoV Research Group. State of knowledge and data gaps of Middle East Respiratory Syndrome Coronavirus (MERS-CoV) in humans. PLoS Curr 2013;5:ecurrents. outbreaks.0bf719e352e7478f8ad85fa30127ddb8. DOI: 10.1371/ currents.outbreaks.0bf719e352e7478f8ad85fa30127ddb8.

6. Lai ST. Treatment of severe acute respiratory syndrome. Eur J Clin Microbiol Infect Dis. 2005 Sep;24(9):583-591. DOI: 10.1007/s10096005-0004-z.

7. Zhao J, Yuan $\mathrm{Q}$, Wang $\mathrm{H}$, et al. Antibody responses to SARS-CoV-2 in patients with novel coronavirus disease 2019. Clin Infect Dis 2020;71(16):2027-2034. DOI: 10.1093/cid/ciaa344.

8. Chen L, Xiong J, Bao L, et al. Convalescent plasma as a potential therapy for COVID-19. Lancet Infect Dis 2020;20(4):398-400. DOI: 10.1016/S1473-3099(20)30141-9.

9. Cheng Y, Wong R, Soo YOY, et al. Use of convalescent plasma therapy in SARS patients in Hong Kong. Eur J Clin Microbiol Infect Dis 2005;24(1):44-46. DOI: 10.1007/s10096-004-1271-9.

10. Gopal BM. Need for more awareness on plasma donation, say doctors. The Hindu. 2020. Available from: https://www.thehindu. $\mathrm{com} /$ news/cities/Visakhapatnam/need-for-more-awareness-onplasma-donation-say-doctors/article32537383.ece.

11. American Red Cross. Convalescent plasma shortage: virus survivors urged to donate plasma to help patients. 2021. Available from: https://www.redcross.org/about-us/news-and-events/news/2020/ covid-19-survivors-urged-to-donate-plasma-to-help-those-battlingcoronavirus.html.

12. Perenc $L$, Pęczkowski R. Knowledge and attitudes of young adults towards donation of COVID-19 convalescent plasma and its therapeutic properties. Research Square 2021. DOI:10.21203/rs.3.rs-286651/v1.

13. Chauhan R, Kumar R, Thakur S. A study to assess the knowledge, attitude, and practices about blood donation among medical students of a medical college in North India. J Family Med Prim Care 2018;7(4):693-697. DOI: 10.4103/jfmpc.jfmpc_54_17.

14. Saeed S. Knowledge, Attitude and practice of plasma donation among COVID-19 recovered patients in Punjab, Pakistan: a crosssectional study. Microbiol Current Res 2021;0(0):10-10. [cited on 30 March 2021]. Available from: https://epidemiology-public health. conference series.com/.

15. Beyene GA. Voluntary blood donation knowledge, attitudes, and practices in Central Ethiopia. IJGM 2020;13:67-76. DOI: 10.2147/IJGM. S246138.

16. Wiwanitkit V. Knowledge about blood donation among a sample of Thai university students. Vox Sanguinis 2002;83:97-99. DOI: 10.1046/j.1423-0410.2002.00209.x.

17. Dubey A, Sonker A, Chaurasia R, et al. Knowledge, attitude and beliefs of people in North India regarding blood donation. Blood Transfus 2014;12(Suppl. 1):s21-s27. DOI: 10.2450/2012.0058-12.

18. Schlumpf KS, Glynn SA, Schreiber GB, et al. Factors influencing donor return. Transfusion 2008;48(2):264-272. DOI: 10.1111/j.15372995.2007.01519.x.

19. Shenga N, Pal R, Sengupta S. Behavior disparities towards blood donation in Sikkim, India. Asian J Transfus Sci 2008;2(2):56-60. DOI: 10.4103/0973-6247.42692.

20. Boulware LE, Ratner LE, Ness PM, et al. The contribution of sociodemographic, medical, and attitudinal factors to blood donation among the general public. Transfusion 2002;42(6):669-678. DOI: 10.1046/j.1537-2995.2002.00120.x.

21. Glynn SA, Williams AE, Nass CC, et al. Attitudes toward blood donation incentives in the United States: implications for donor recruitment. Transfusion 2003;43(1):7-16. DOI: 10.1046/j.1537-2995.2003. 00252.x. 